\title{
Competitive Exclusion and Evolution: Convergence Almost Never Produces Ecologically Equivalent Species
}

\author{
(A Comment on McPeek, "Limiting Similarity? The Ecological Dynamics of Natural Selection \\ among Resources and Consumers Caused by Both Apparent and Resource Competition")
}

\author{
Liz Pásztor, ${ }^{1, \star}$ György Barabás, ${ }^{2,3}$ and Géza Meszéna ${ }^{3,4}$ \\ 1. Department of Genetics, Eötvös Loránd University (ELTE), Budapest, Hungary; and Hungarian Academy of Sciences (MTA) Centre for \\ Ecological Research, Tihany H-8237 Klebelsberg Kuno u. 3, Budapest, Hungary; 2. Division of Theoretical Biology, Department of Physics, \\ Chemistry, and Biology (IFM), Linköping University, Linköping, Sweden; 3. MTA-ELTE Theoretical Biology and Evolutionary Ecology \\ Research Group, ELTE, Budapest, Hungary; 4. Department of Biological Physics, ELTE, Budapest, Hungary
}

Submitted July 22, 2019; Accepted November 11, 2019; Electronically published February 28, 2020

\begin{abstract}
AвSTRACT: In a recent modeling study ("Limiting Similarity? The Ecological Dynamics of Natural Selection among Resources and Consumers Caused by Both Apparent and Resource Competition") that appeared in the April 2019 issue of The American Naturalist, Mark A. McPeek argued that ecologically equivalent species may emerge via competition-induced trait convergence, in conflict with naive expectations based on the limiting similarity principle. Although the emphasis on the possibility of the convergence of competitors is very timely, here we show that the proposed mechanism will only lead to actual coexistence in the converged state for specially chosen fine-tuned parameter settings. It is therefore not a robust mechanism for the evolution of ecologically equivalent species. We conclude that invoking trait convergence as an explanation for the co-occurrence of seemingly fully equivalent species in nature would be premature.
\end{abstract}

Keywords: neutral species, competition, adaptive dynamics, cryptic species, character displacement, coexistence theory.

Niche segregation and neutrality - or perhaps the continuum between these two extremes - have been considered as possible explanations of species co-occurrence (Holt 2006; Adler et al. 2007; Rapacciuolo and Blois 2019). While there are strong theoretical arguments (Chesson 1991) and ample empirical evidence (Janzen et al. 2017) for niche segregation, there are also several observations of the co-occurrence of reproductively isolated species with no obvious ecological differentiation between them (McPeek and Gomulkiewicz 2005). In a recent article, McPeek (2019)

* Corresponding author; email: lizpasztor@gmail.com.

ORCIDs: Pásztor, https://orcid.org/0000-0003-2630-5673; Barabás, https:// orcid.org/0000-0002-7355-3664; Meszéna, https://orcid.org/0000-0002-6557 -8423 .

Am. Nat. 2020. Vol. 195, pp. E112-E117. (C) 2020 by The University of Chicago. 0003-0147/2020/19504-59381\$15.00. All rights reserved. DOI: $10.1086 / 707610$ investigates the possibility of the emergence of neutral coexistence via convergent evolution besides discussing opportunities for divergence. McPeek emphasizes that his results contrast with expectations from naive versions of the limiting similarity principle and argues for supplementing the purely ecological study of conditions for coexistence with the explicit modeling of evolutionary dynamics. This is an important point, and McPeek (2019) makes a great contribution by emphasizing it in a remarkably clear way. However, we feel compelled to clarify how the principle of limiting similarity in fact still applies to McPeek's conclusions, not only because a series of our own work was cited as supporting the naive expectations but also because of the central role played by this principle in coexistence theory. We agree with McPeek that the co-occurrence of identical species differing only in neutral traits is a theoretical possibility. However, we wish to show that competition-driven convergence to neutrality will happen only under highly restrictive conditions. It is therefore unlikely to be an explanation for the longterm co-occurrence of competing cryptic or sister species. Hence, we wish to clarify the relationship between robustness of coexistence and similarity, to specify the relevance of the principle of weak limiting similarity (Meszéna et al. 2006; Burgess et al. 2019) for McPeek's (2019) model via simulations of evolutionary trajectories, and to discuss the problem of convergence within the universal framework of adaptive dynamics. At the end we summarize the conclusions for the emergence of species.

\section{Robustness and the Weak Limiting Similarity Principle \\ Competition-driven convergence seems to contradict the principle of limiting similarity. If such convergence leads}


to completely identical species whose relative densities fluctuate via ecological drift, their long-term coexistence is expected as long as their population sizes are sufficiently large (McPeek and Gomulkiewicz 2005). Such neutrality is robust in the face of environmental disturbances, as the environmental response functions of two biologically identical species are also identical. Thus, two completely identical species may coexist, and their coexistence is robust to environmental disturbances. Robustness of a system's behavior to parametric uncertainties is an important concept, not only for niche theory (Chesson 1991) but also for systems biology - for example, in the context of morphogenesis (Eldar et al. 2004), in metabolic and gene regulatory networks (Nijhout et al. 2019), and in engineering (Aström and Murray 2008). In general, robustness is a consequence of differences between negative feedback loops. Too much similarity in feedback structure results in diminished robustness. The weak limiting similarity principle claims that the coexistence of a given set of (nonidentical) populations is restricted to a narrow range of the externally determined parameters when the overlap is large between either the "impact" niche or the "sensitivity" niche of the populations (Meszéna et al. 2006; called the "principle of robust coexistence" in Pásztor et al. 2016). In the context of MacArthur and Levins (1967), coexistence is sufficiently robust when the competing species are roughly separated by the niche width defined by the resource utilization functionotherwise, coexistence becomes very sensitive to parameter perturbations (Szabó and Meszéna 2006). As a consequence, while two identical species may coexist, any difference between their functional traits makes their coexistence prone to changes in the external environment. Moreover, random genetic mutations turn the coexistence of two identical species structurally unstable in the long run: a mutation providing a slight difference in fitness will lead to competitive exclusion.

As McPeek (2019) correctly emphasizes, the validity of the weak limiting similarity principle does not guarantee divergent evolution. This purely ecological principle assumes fixed species. Still, it does apply at each step of any evolutionary process. When two species converge driven by competition, their coexistence becomes less and less robust in the face of environmental disturbances because of increasing similarity of their niches. We show below that by considering the robustness of coexistence, it becomes clear that reaching neutral coexistence by convergence is extremely unlikely in noisy systems.

\section{Unrobust Evolutionary Trajectories in McPeek's Model}

Trait convergence or divergence are both natural outcomes in McPeek's model. Whether species competing for resources ultimately converge or diverge may seem difficult to discern. In fact, it is easier than it seems at first sight. Evolving away from consuming an already heavily exploited resource is advantageous only if a different resource is available and the diet change does not incur large fitness costs. Assume that two species with widely different diets arrive in a habitat with narrow resource diversity. They will evolve convergently, as both species will adapt to the resource distribution existing in the new habitat. Convergent evolution either leads to their coexistence with smaller diet differentiation or to one of them being lost via the strengthening competition (Germain et al. 2018).

McPeek's model considers two resource and two consumer populations, each characterized by the phenotypic distribution (mean and variance) of a quantitative trait. Interactions between the species induce frequency-dependent selection on the means of these distributions. The instantaneous per capita population growth rates define the population fitness values, which are in turn composed of the contributions from all phenotypes within those populations. Trait evolution is governed by quantitative genetic rules (Taper and Case 1985) while the ecological dynamics bears most resemblance to MacArthur's (1972) model of resource competition, due to a self-regulation term that effectively makes resource growth logistic in the absence of consumption (this also means that resource supply is not modeled as in Tilman 1982, with biotic resources assumed instead). The model is similar in spirit to quantitative genetic models of competition along a trait axis, where the more similar the consumers are in their trait, the more similarly they consume the resources and so the more they compete (Slatkin 1980; Taper and Case 1985; Schreiber et al. 2011; Vasseur et al. 2011; Barabás and D’Andrea 2016). The difference is that in the model of McPeek, the resource populations themselves may evolve to provide a better or worse match with the traits of their consumers.

Focusing here on the case of convergence, figure $1 \mathrm{~A}$ reproduces one such scenario, taken straight from McPeek (2019). The dynamics end up with the co-occurrence of two ecologically identical species. As mentioned before, however, such coexistence is not robust: even the smallest difference in any fitness-affecting parameter may lead to competitive exclusion. Accordingly, figure $1 B$ presents a case where the environmental component of consumer 2's phenotypic variance is slightly increased, thus making the two species slightly different; coexistence is lost, and consumer 1 wins. In figure $1 C$, the two consumers share identical parameters, but consumer 2 gets a head start in the race toward the optimal phenotype by being closer to it initially than its competitor. Consumer 2 is excluded again before it reaches the optimum. We argue below that these results represent the generic case: trait convergence requires both perfectly identical parameters between the competitors and highly symmetrical initial conditions. 

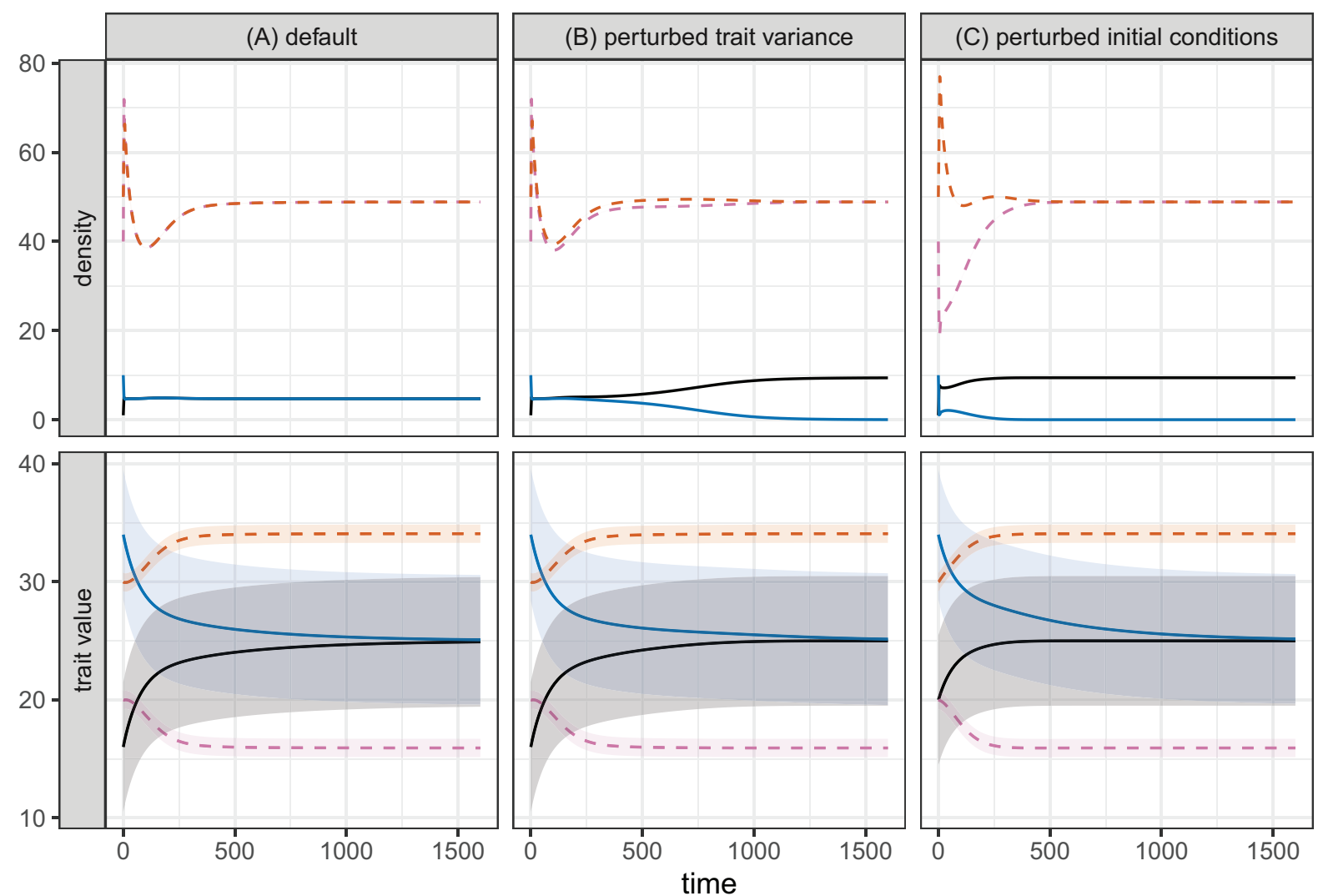

Figure 1: Numerical illustration that convergence to ecologically equivalent species in the model of McPeek (2019) is oversensitive to both perturbing parameters and initial conditions. Top and bottom rows show population densities and trait values, respectively, over time. Solid lines are consumers, and dashed lines are resources; different colors stand for different species. In the bottom row, lines show trait means, and the shaded areas around them show the $1 \sigma$ trait standard deviations. In the default scenario $(A)$, parameters are $c_{0 i}=3, d_{i}=0.02$, $\gamma_{i}=0.01, z_{N_{i}}^{f}=25, G_{R_{i}}=0.2, E_{R_{i}}=0.4, G_{N_{i}}=0.2, E_{N_{i}}=30, a_{0 i}=0.5, b_{i}=0.1, \beta_{i}=5.0, f_{i}=0.15, g_{i}=0, \theta_{i}=0.01, f_{0 i}=1.2$, $z_{R_{1}}^{c}=20$, and $z_{R_{2}}^{c}=30$, with initial conditions $R_{1}(0)=40, R_{2}(0)=50, N_{1}(0)=1, N_{2}(0)=10, z_{R_{1}}(0)=20, z_{R_{2}}(0)=30, z_{N_{1}}(0)=16$, and $z_{N_{2}}(0)=34$. In this scenario, the consumers converge in their mean trait, effectively becoming identical (neutral) species. In the second scenario $(B)$, everything is as before except that $E_{N_{2}}$ is increased from 30 to 31. This small perturbation of the trait variance means that although the consumers still converge in their mean traits, consumer 2 is now excluded. The third scenario $(C)$ is like the default scenario $(A)$ except that $z_{N_{1}}(0)$ is changed from 16 to 20 . This brings the first consumer's initial mean trait closer to the middle, enabling it to get to the evolutionary optimum quicker than consumer 2. This initial asymmetry in starting conditions is sufficient to exclude consumer 2 while it is evolving toward the middle.

The theory of adaptive dynamics (Metz et al. 1996; Geritz et al. 1997, 1998; Metz 2012) provides a general framework for understanding the evolutionary trajectories of continuous character(s), taking frequency-dependent selection into account but without considering the complications of diploid genetics. McPeek's model fits into this framework. According to the theory, continuous directional evolution proceeds in the direction of increasing fitness in each generation, eventually arriving at a singular point where the fitness gradient becomes zero. Frequency dependence manifests itself in the fact that the convergencestable singular point to which directional evolution converges is not necessarily a fitness maximum. Whether it is a fitness maximum or a fitness minimum corresponds to the cases of converging or diverging evolution, respectively.
Diverging evolution is neither the only option nor a priori more likely than convergence within this framework.

Figure 2 presents the same results as figure 1 using mutual invasibility plots (Brännström et al. 2013). In these plots the regions of coexistence/co-occurrence are plotted as a function of the evolving traits of the two consumers with fixing resource traits at their evolutionarily stable outcomes (this state is independent of the competitive outcome between the two consumers; see fig. 1). Figure $2 A$ corresponds to the two cases with the same parameter values given in figure $1 A$ and $1 C$. The region of coexistence has a single point on the main diagonal, meaning that coexistence of identical species is possible but only at that single common mean trait value, which is the singular point of adaptive dynamics. The solid arrow within the coexistence 

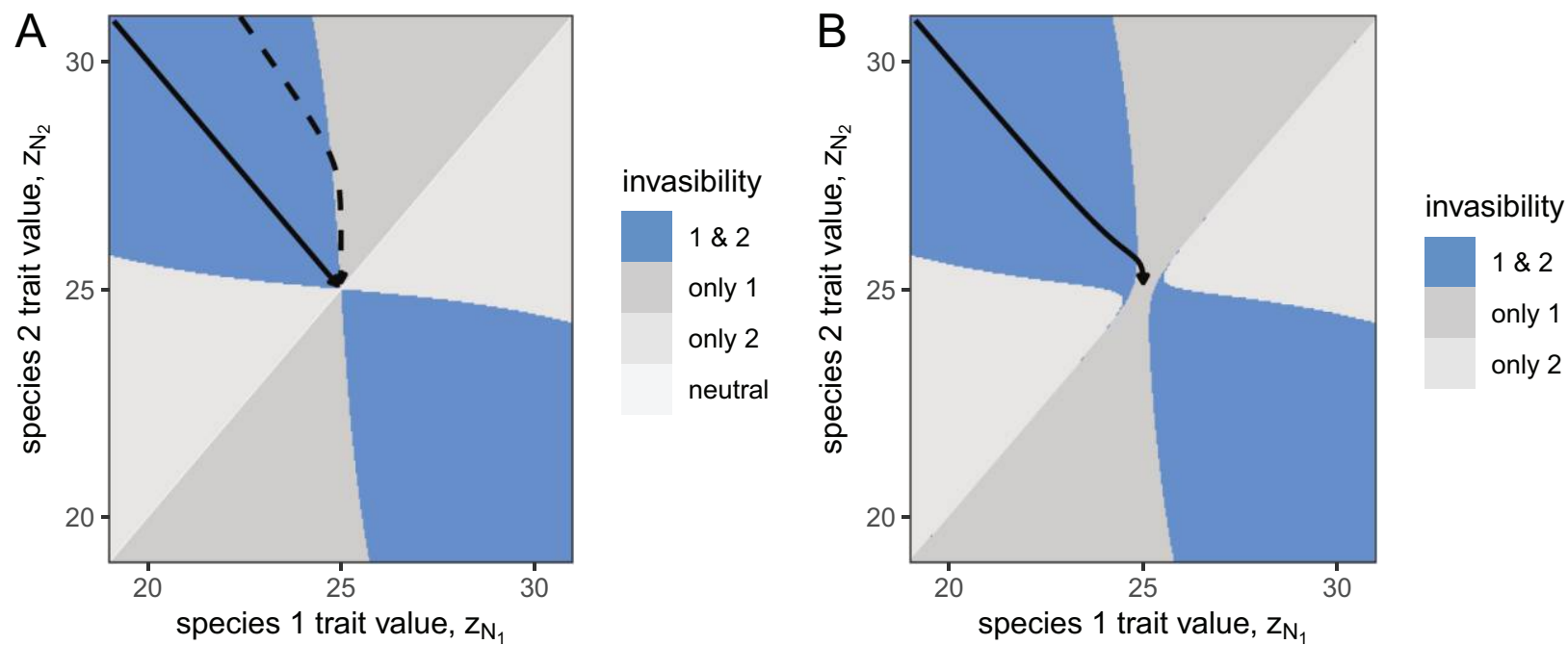

Figure 2: Mutual invasibility plots in McPeek's (2019) model. A, Parameters are as in the default scenario of figure 1. Resource mean phenotypes are fixed at $z_{R_{1}}=15.9$ and $z_{R_{2}}=34.1$, the evolutionarily stable outcomes of the same scenario. Mean trait values are shown for consumer species 1 (abscissa, $z_{N_{1}}$ ) and species 2 (ordinate, $z_{N_{2}}$ ). The blue region shows trait combinations of mutual invasibility, where each species can invade, in turn, the monoculture equilibrium of its competitor. Outside this region, either species 1 (dark gray) or species 2 (light gray) wins, and there is no coexistence or there is neutrally stable coexistence (white strip along identical trait values). Arrows show the dynamical trajectories of consumers' trait means from figure $1 A$ (solid) and $1 C$ (dashed). In the latter scenario, the trajectory moves outside the region of mutual invasibility, driving species 2 extinct. $B$, As before, but with $E_{N_{2}}$ increased from 30 to 31 (as in fig. 1B). The strip of neutral coexistence has disappeared; instead, for $z_{N_{1}}=z_{N_{2}}$, it is always species 1 that wins. Therefore, even though evolution still drives the system toward the evolutionarily stable point in the middle, coexistence is no longer possible at this point. In summary, while convergence of traits is a natural and robust outcome in this model, it does not guarantee co-occurrence - in fact, both model parameters and initial conditions must be carefully fine-tuned to avoid competitive exclusion.

region represents the evolutionary trajectory when both the parameters and the initial conditions of the two consumers are identical, as in figure $1 \mathrm{~A}$. It reaches the co-occurrence point on the main diagonal. The evolutionary trajectory shown by the dashed arrow corresponds to the different initial conditions of figure $1 C$. It moves out of the coexistence region and remains outside, causing species 2 to become extinct before reaching the common point of convergence. Figure $2 B$ depicts the case given in figure $1 B$. When a parameter is different for the two consumers, the region of coexistence does not include the main diagonal. Therefore, coexistence of two different consumers with identical trait distributions (i.e., without niche segregation) is not possible.

The sharp shape of the coexistence range in the vicinity of the singular point is general, as it is the geometric consequence of superimposing two pairwise invasibility plots (see fig. 3 in Geritz et al. 1998). Therefore, it is a general conclusion that converging evolution results in co-occurrence only in an unlikely fine-tuned case. McPeek's results on the convergence to neutral species thus demonstrate exceptional nongeneric cases, when a fitness maximum is approached from the same distance, the fitness of the compet- ing species is the same at each step, and a continuous path to the singular point does exist. In this way, the weak limiting similarity theorem is valid at each step on the way to the singular point, assuming a separation of ecological and evolutionary timescales. (Without such separation, as in McPeek's model, sufficiently fast evolution may still reach the singular point, though with potentially very low density of the inferior species.) However, even these cases are highly special, as we have to assume that the only difference between the species is in the evolving trait(s). Otherwise, one species will disappear when the trait difference becomes smaller than the threshold set by other (parameter) differences between the species.

\section{Conclusions}

The vast number of co-occurring, seemingly identical, ecologically equivalent sister and cryptic species provide empirical grounds for challenging conventional ecological wisdom, supporting the view that these species have equal fitness in any environment, with ecological drift determining their fate instead of stabilizing frequencydependent interactions (McPeek and Gomulkiewicz 2005; 
Leibold and McPeek 2006). Certainly, this type of coexistence is a theoretical possibility, as extinction by drift is a very slow process in large populations (Hubbell 2001).

Then the question arises: Is there an evolutionary mechanism that can produce such cryptic species? Immediate emergence of cryptic, neutral species does not seem plausible. For example, allopolyploid hybrid speciation leads to immediate reproductive isolation but also with differences in fitness components (Taylor and Larson 2019). In addition, there are indications that resource partitioning among larvae may maintain the coexistence of cryptic lepidopteran species (Janzen et al. 2017) and of Müllerian mimics of passion vine butterflies (Benson 1978).

Emergence of neutral species purely by way of sexual selection is also considered unlikely, in both allopatry and sympatry (Servedio and Boughman 2017; Kopp et al. 2018). This is because rare variants, which mate more successfully between themselves than with other variants or choose extreme partners, have lower fitness just because they are rare (Arnegard and Kondrashov 2004). This disadvantage must be compensated with some frequencydependent advantage, stabilizing their coexistence with more common variants. To our knowledge, it is yet to be demonstrated that mutations accumulated in allopatric populations provide only reproductive isolation without affecting fitness (Toews et al. 2016).

In light of these difficulties, the emergence of ecologically equivalent but reproductively isolated species by convergent evolution is an intriguing suggestion. However, as we demonstrated above, this process is extremely unlikely, as avoidance of competitive exclusion during the process depends on a precise fine-tuning of parameters and initial conditions.

While it is difficult to imagine the emergence of strictly neutral but reproductively isolated cryptic species, there is strong support for the opposite: gene flow between ecologically different species (Nosil 2012; Taylor and Larson 2019). Considering all of these arguments and given the necessary gaps in our empirical knowledge, we still raise the question of whether hidden species differences, generating frequency-dependent ecological dynamics, may not be the rule rather than the exception in explaining the coexistence of cryptic species.

\section{Acknowledgments}

We thank Mark McPeek, Éva Kisdi, Hans Metz, and Bob Holt for comments on the manuscript. Suggestions by Daniel Bolnick, Joachim Hermisson, Peter Chesson, and an anonymous referee all helpfully improved our presentation. L.P. and G.M. were funded by the Hungarian National Research, Development, and Innovation Office (grant NKFI-123796). L.P. was also funded by GINOP-
2.3.2-15-2016-00057. G.B. was funded by the Swedish Research Council (grant VR 2017-05245).

\section{Literature Cited}

Adler, P. B., J. HilleRisLambers, and J. M. Levine. 2007. A niche for neutrality. Ecology Letters 10:95-104.

Arnegard, M. E., and A. S. Kondrashov. 2004. Sympatric speciation by sexual selection alone is unlikely. Evolution 58:222-237.

Aström, K. J., and R. M. Murray. 2008. Feedback systems: an introduction for scientists and engineers. Princeton University Press, Princeton, NJ.

Barabás, G., and R. D’Andrea. 2016. The effect of intraspecific variation and heritability on community pattern and robustness. Ecology Letters 19:977-986.

Benson, W. W. 1978. Resource partitioning in passion vine butterflies. Evolution 32:493-518.

Brännström, Å., J. Johansson, and N. Von Festenberg. 2013. The hitchhiker's guide to adaptive dynamics. Games 4:304-328.

Burgess, M. G., A. Fredston-Hermann, D. Tilman, M. Loreau, and S. D. Gaines. 2019. Broadly inflicted stressors can cause ecosystem thinning. Theoretical Ecology 12:1-17.

Chesson, P. 1991. A need for niches? Trends in Ecology and Evolution 6:26-28.

Eldar, A., B.-Z. Shilo, and N. Barkai. 2004. Elucidating mechanisms underlying robustness of morphogen gradients. Current Opinion in Genetics and Development 14:435-439.

Geritz, S. A. H., É. Kisdi, G. Meszéna, and J. A. J. Metz. 1998. Evolutionary singular strategies and the adaptive growth and branching of evolutionary trees. Evolutionary Ecology 12:35-57.

Geritz, S. A. H., J. A. J. Metz, É. Kisdi, and G. Meszéna. 1997. Dynamics of adaptation and evolutionary branching. Physical Review Letters 78:2024-2027.

Germain, R. M., J. L. Williams, D. Schluter, and A. L. Angert. 2018. Moving character displacement beyond characters using contemporary coexistence theory. Trends in Ecology and Evolution 33:74-84.

Holt, R. D. 2006. Emergent neutrality. Trends in Ecology and Evolution 21:531-533.

Hubbell, S. P. 2001. The unified neutral theory of biodiversity and biogeography. Princeton University Press, Princeton, NJ.

Janzen, D. H., J. M. Burns, Q. Cong, W. Hallwachs, T. Dapkey, R. Manjunath, M. Hajibabaei, P. D. Hebert, and N. V. Grishin. 2017. Nuclear genomes distinguish cryptic species suggested by their DNA barcodes and ecology. Proceedings of the National Academy of Sciences of the USA 114:8313-8318.

Kopp, M., M. R. Servedio, T. C. Mendelson, R. J. Safran, R. L. Rodríguez, M. E. Hauber, E. C. Scordato, L. B. Symes, C. N. Balakrishnan, and D. M. Zonana. 2018. Mechanisms of assortative mating in speciation with gene flow: connecting theory and empirical research. American Naturalist 191:1-20.

Leibold, M. A., and M. A. McPeek. 2006. Coexistence of the niche and neutral perspectives in community ecology. Ecology 87:13991410 .

MacArthur, R. H. 1972. Geographical ecology: patterns in the distribution of species. Princeton University Press, Princeton, NJ.

MacArthur, R. H., and R. Levins. 1967. Limiting similarity, convergence, and divergence of coexisting species. American Naturalist 101:377-385. 
McPeek, M. A. 2019. Limiting similarity? The ecological dynamics of natural selection among resources and consumers caused by both apparent and resource competition. American Naturalist 193:E92-E115

McPeek, M. A., and R. Gomulkiewicz. 2005. Assembling and depleting species richness in metacommunities: insights from ecology, population genetics, and macroevolution. Pages 355-373 in M. Holyoak, M. A. Leibold, and R. D. Holt, eds. Metacommunities. University of Chicago Press, Chicago.

Meszéna, G., M. Gyllenberg, L. Pásztor, and J. A. J. Metz. 2006. Competitive exclusion and limiting similarity: a unified theory. Theoretical Population Biology 69:68-87.

Metz, J. A. J. 2012. Adaptive dynamics. Pages 1-17 in A. Hastings and L. J. Gross, eds. Encyclopedia of Theoretical Ecology. University of California Press, Berkeley.

Metz, J. A. J., S. A. H. Geritz, G. Meszéna, F. J. A. Jacobs, and J. S. van Heerwaarden. 1996. Adaptive dynamics: a geometrical study of the consequences of nearly faithful reproduction. Pages 183231 in S. J. van Strien and S. M. Verduyn Lunel, eds. Stochastic and spatial structures of dynamical systems. Proceedings of the Royal Dutch Academy of Science, Amsterdam.

Nijhout, H. F., J. A. Best, and M. C. Reed. 2019. Systems biology of robustness and homeostatic mechanisms. Wilev Interdisciplinary Reviews: Systems Biology and Medicine 11:e1440.

Nosil, P. 2012. Ecological speciation. Oxford University Press, Oxford.

Pásztor, L., Z. Botta-Dukát, G. Magyar, T. Czárán, and G. Meszéna. 2016. Theory-based ecology: a Darwinian approach. Oxford University Press, Oxford.

Rapacciuolo, G., and J. L. Blois. 2019. Understanding ecological change across large spatial, temporal, and taxonomic scales: in- tegrating data and methods in light of theory. Ecography 42:120.

Schreiber, S. J., R. Bürger, and D. I. Bolnick. 2011. The community effects of phenotypic and genetic variation within a predator population. Ecology 92:1582-1593.

Servedio, M. R., and J. W. Boughman. 2017. The role of sexual selection in local adaptation and speciation. Annual Review of Ecology, Evolution, and Systematics 48:85-109.

Slatkin, M. 1980. Ecological character displacement. Ecology 6:163177.

Szabó, P., and G. Meszéna. 2006. Limiting similarity revisited. Oikos 112:612-619.

Taper, M. L., and T. J. Case. 1985. Quantitative genetic models for the coevolution of character displacement. Ecology 66:355-371.

Taylor, S. A., and E. L. Larson. 2019. Insights from genomes into the evolutionary importance and prevalence of hybridization in nature. Nature Ecology and Evolution 3:170.

Tilman, D. 1982. Resource competition and community structure. Princeton University Press, Princeton, NJ.

Toews, D. P., S. A. Taylor, R. Vallender, A. Brelsford, B. G. Butcher, P. W. Messer, and I. J. Lovette. 2016. Plumage genes and little else distinguish the genomes of hybridizing warblers. Current Biology 26:2313-2318

Vasseur, D. A., P. Amarasekare, V. H. W. Rudolf, and J. M. Levine. 2011. Eco-evolutionary dynamics enable coexistence via neighbordependent selection. American Naturalist 178:E96-E109.

Associate Editor: Joachim Hermisson Editor: Daniel I. Bolnick

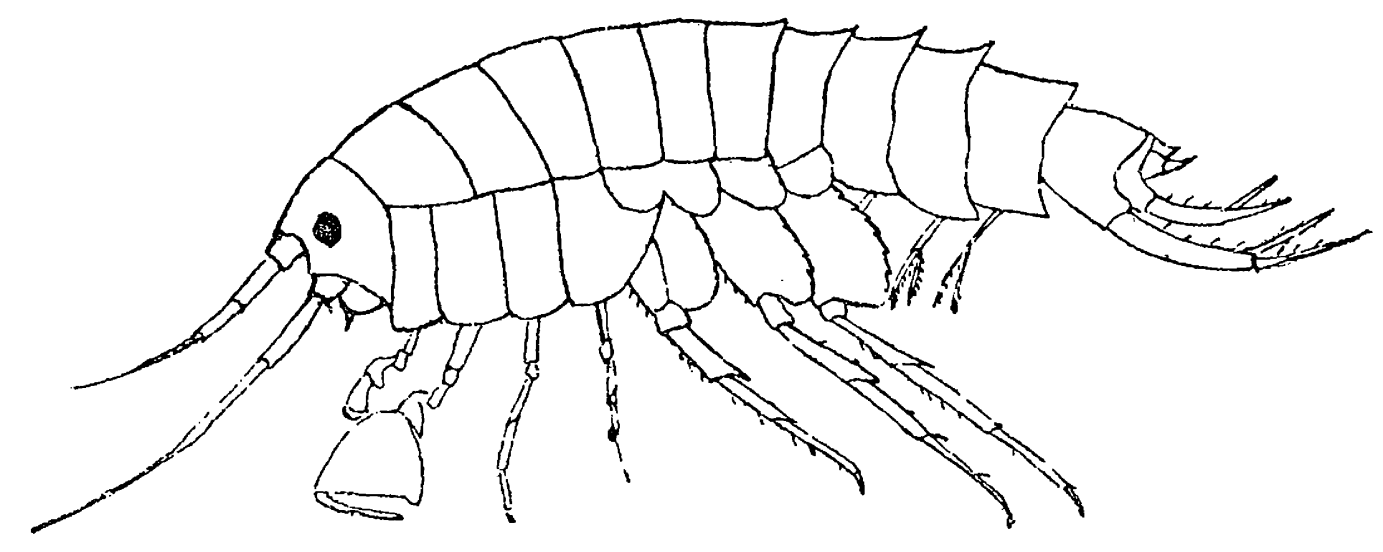

"The marine species usually inhabit the shores above low-water mark, and the previously described freshwater species are found in the shallow water of brooks, pools, or edges of lakes." Figured: "Allorchestes latimanus." From the review of "The Crustacea of Lake Titicaca" (The American Naturalist, 1877, 11:116-118). 